\title{
Solar drying of granulated waste blends for dry biofuel production
}

\author{
Małgorzata Wzorek ${ }^{1}$
}

Received: 25 November 2020 / Accepted: 3 February 2021 / Published online: 26 February 2021

(C) The Author(s) 2021

\begin{abstract}
In the paper, results of drying biofuels from sewage sludge using solar energy are presented. Drying rates of biofuels made from sewage sludge and coal slime (PBS), sewage sludge and meat and bone meal (PBM), and sewage sludge and sawdust (PBT) with $15-\mathrm{mm}$ and 35-mm granule particle size were studied. Tests were performed in a solar greenhouse dryer equipped with a specially designed mixing system. Experiments were aimed at determining the drying time of biofuels under various weather conditions in the southwestern part of Poland. In summer, in order to determine the best conditions for drying, tests were performed using various parameters, i.e., layers of various thickness, such as 5,10, and $20 \mathrm{~cm}$, and various mixing intensity (no mixing, mixing 3 and 5 times/day). In spring and the fall, 10-cm thick layers combined with 5 times mixing of fuels per day were used. The performed tests demonstrated that it is beneficial to dry fuels in 10-cm thick layer. In spring and the fall, PBS and PBM biofuels laid out in layers with just such thickness showed moisture content reduced to less than $10 \%$ after 8 days, while the PBT biofuel reached the same level after 14 days. In summer, the same result may be obtained for all the biofuels after 4 days on average. The presented original method of solar drying of biofuels obtained from sewage sludge and other waste may be used in wastewater treatment plants which process sewage sludge into fuels without incurring any additional costs for supplying heat.
\end{abstract}

Keywords Solar energy $\cdot$ Drying $\cdot$ Greenhouse $\cdot$ Sewage sludge $\cdot$ Biofuel $\cdot$ Waste

\section{Introduction}

Sewage sludge management is one of the significant challenges of wastewater management in Poland. Sewage sludge represents a major type of waste produced by wastewater treatment plants and accounts for roughly $1-2 \%$ of the total amount volume of wastewater treated. Its disposal can add significant energy demand to the already high requirements of a treatment plant (Capodaglio and Olsson 2020). An amount of sewage sludge cannot be prevented and is disposed in line with the requirements regarding the quality of treated sewage.

A total of 3253 municipal wastewater treatment plants were operating in Poland in 2019, which is almost three times as many when compared to 1995 , serving more than 28.5 million

Responsible Editor: Philippe Garrigues

Małgorzata Wzorek

m.wzorek@po.edu.pl

1 Department of Process and Environmental Engineering, Faculty of Mechanical Engineering, Opole University of Technology, ul. Mikołajczyka 5, 45-271 Opole, Poland residents (i.e., $73.5 \%$ of the total population (Statistics Poland 2018).

Wastewater treatment plants use ever more efficient treatment systems, such as biological treatment with extended nutrients removal (three-phase and double-phase, hybrid, cyclical, semi-cyclical, or single-phase systems with chemical phosphorus precipitation). The resulting relationship is quite simple - the more sophisticated waste treatment technologies, the more sewage sludge is generated which is additionally harder to dispose of.

According to data published by Statistics Poland (2018), $613,000 \mathrm{t}$ of dry mass of sewage sludge were produced in Poland by municipal wastewater treatment plants in 2016, additionally around $11,000 \mathrm{t}$ were generated by domestic sewage treatment systems in rural areas (Pawlita-Posmyk and Wzorek 2016).

The amount of generated sewage sludge depends on many factors, mainly on the content of organic pollutants in the sludge and - as mentioned earlier - on the technology of its treatment.

The varying composition and amounts of micropollutants in the sewage sludge, such as polycyclic aromatic hydrocarbons (PAHs), heavy metals, polychlorinated biphenyls (PCBs), and pathogens make the sludge management 
problems difficult to solve (Ozaki et al. 2017; Mailler et al. 2014; Raheem et al. 2018; Pawlita-Posmyk and Wzorek 2017). A major contributing parameter here is attributable to the high degree of sludge hydration which in the case of raw sludge amounts to more than $99 \%$, with between 80 and $65 \%$ for mechanically dehydrated sludge.

One of the methods applied to significantly reduce both the mass and volume of sewage sludge involves thermal processes, such as combustion, pyrolysis, or gasification (Fonts et al. 2012; Oladejo et al. 2019). Pyrolysis and gasification, in addition to mass reduction, can also generate liquid or gaseous fuels for subsequent use (Capodaglio et al. 2016); these can be of particular economic interest in view of a recent EU transportation policy (Raboni et al. 2015).

The current role and immediate prospects of biofuels production from waste products including sewage sludge, wood and coal residues, and other organic waste are the topic of intensive research today (Callegari et al. 2020).

However, the fundamental applicability of sewage sludge in the energy-rich biofuels generation process is associated with the necessity of fulfillment of requirement of low moisture content.

Sewage sludge containing 20-30\% of dry mass may only be incinerated after another fuel has been added, while autothermal combustion will occur only after the mass has been partially dried up to 50\% (Werther and Ogada 1999). Drying the sewage sludge up to roughly $10 \%$ of dry mass makes it suitable for application in coal co-combustion processes, such as power and heat plants and waste incineration plants or in the process of cement kiln production (Murakami et al. 2009; Wasielewski et al. 2013; Wzorek and Troniewski 2007).

Sewage sludge constitutes the type of waste for which the boundary value of mechanical dehydration is determined with the content of wastewater solids in the range of $30 \div 35 \%$. The higher degree of dehydration, equal to $90 \%$, may only be achieved through thermal drying. This implies that a substantial amount of water must be evaporated, which is energetically expensive and could offset the advantages of energetic recovery from the combustion.

\section{Drying of biosolids: thermal vs. solar}

Thermal drying is frequently used in the processing of biomass and waste into fuels: lowering the moisture content improves their energy characteristics - improvement of the low heating value and acquiring physical properties that results in lower transport and warehousing costs. In the case of organic waste, drying is also used to improve hygienization of the product; however, the process contributes to higher production costs.

Owing to the ever more popular application of waste, biomass, and sewage sludge, an increased interest in the study of drying has been observed, with high-temperature drying processes (Collard et al. 2017; Perazzini et al. 2016), biological drying (Ab Jalil et al. 2016; Zhang et al. 2018), and the potential for using alternative sources of energy to this end being considered (Singh et al. 2018; Maurer and Müller 2019).

The conventional processes of drying require a considerable amount of energy. For example, in the case of sewage sludge, and depending on the drying method, the water evaporation may require to either $0.7-0.9 \mathrm{kWh} / \mathrm{kg}$ or even with the expected energy consumption ratio to be of up to $1.4 \mathrm{kWh} / \mathrm{kg}$ (Bennamoun et al. 2013; Tańczuk and Kostowski 2021).

An alternative to the thermal drying methods is drying with the application of solar energy. Literature on the subject provides extensive information about the research into the application of solar energy to dry agricultural crops and wood both in solar-drying plants and outdoors (VijayaVenkataRaman et al. 2012; Campean and Marinescu (2011) and recently, also to dry waste, biomass, and sewage sludge (Tun and Juchelková 2019; Alamia et al. 2015; Mehrdadi et al. 2007; Bennamoun 2012).

In Europe, a number of solar sewage sludge dryers have been constructed, mainly in Germany, France, Austria, and Switzerland, but also in Poland (I IST-Anlagenbau GmbH 2020; Thermo-system. Industrie-\&Trocknungstechnik GmbH 2020; Bożym and Bok 2015).

Drying technologies based on solar energy in solar dryers utilize the greenhouse effect produced inside a structure covered with a sunlight-transmitting material.

Solar dryers used to dry sewage sludge feature a greenhouse structure covered with glass, foil, or polycarbonate panels (Singh et al. 2018).

The drying rate depends on a number of factors, including weather, such as the temperature and relative humidity of the air, solar radiation intensity, the heating medium flow direction and speed, the size of the uncovered area of the material to be dried, and the bed thickness (Luboschik 1999; Yoo et al. 2017; Hii et al. 2012).

One of the key parameters of the drying process consists in the relative humidity of the drying air. The lower the relative humidity of the drying air, the more water evaporates from the product, ensuring a lower moisture content of the final product (Helwa et al. 2004). Shin et al. (2000) indicate that greater influence of drying rate is presented by relative humidity instead of its temperature. Luboschik (1999) has estimated that as long as the partial pressure of water vapor in the air depends only on the absolute humidity rather than the air temperature, the best results are obtained by drying with the warm sludge and dry air. For those reasons, solar dryers feature exchange air systems, such as air supply and air exhaust.

Undoubtedly, solar irradiance represents a most important factor in the solar drying since it is the basic source of solar energy required to dry the material at hand.

The prevailing weather conditions in Poland favor utilizing the solar energy available. The strategy for using renewable 
energy resources even assumes the technical potential for using solar radiation energy may reach 1340 PJ (Polish's energy policy until 20302009 ).

At this latitude, the average annual irradiation time equals roughly $1600 \mathrm{~h}$ while the annual solar radiation density ranges from 956 to $1100 \mathrm{kWh} / \mathrm{m}^{2}$ (EU Science Hub 2020). The 24-h average distribution of solar radiation intensity for Opole (southwestern part of Poland) is presented in Fig. 1.

Another factor that increases contact between the material to be dried and the drying medium is the mixing system. Various types of mixing equipment are used to dry the sewage sludge only, such as chain or shovel turners, electric moles, or a prism system for turning the sludge.

The systems also call for a different thickness of the material to be dried which — in the case of Thermo-system ${ }^{\circledR}$ — varies from 1 to $10 \mathrm{~cm}$ (Thermo-system); for the Wende Wolf®, it is between 30 and $40 \mathrm{~cm}$ (IST-Anlageenbau $\mathrm{GmbH}$ ); and in the case of prism technologies, it may even be as high as $50 \mathrm{~cm}$ (Trojanowska 2016).

In Europe, solar dryers mostly start operating in spring, continue in summer, and close down in the fall. Only those plants which have been additionally equipped with an underfloor heating installation can operate in winter. The evaporating efficiency factor of the solar dryers ranges from 590 to $792 \mathrm{~kg}_{\mathrm{H} 2 \mathrm{O} / \mathrm{m}^{2}}$ a under the conditions prevailing in Poland; while in the case of hybrid dryers (with an additional source of heat), it stands at $856-927 \mathrm{~kg}_{\mathrm{H} 2 \mathrm{O}} / \mathrm{m}^{2} \cdot \mathrm{a}$ (Trojanowska 2016).

The technologies currently available in the market which mix the sludge during the drying in solar dryers are used to dry the sewage sludge only, a process that delivers dry granulate of up to $5 \mathrm{~mm}$ in size and 10-20\% moisture content.

Depending on its calorific value ( $>12 \mathrm{MJ} / \mathrm{kg})$, dried sludge may be used for combustion processes, among others.

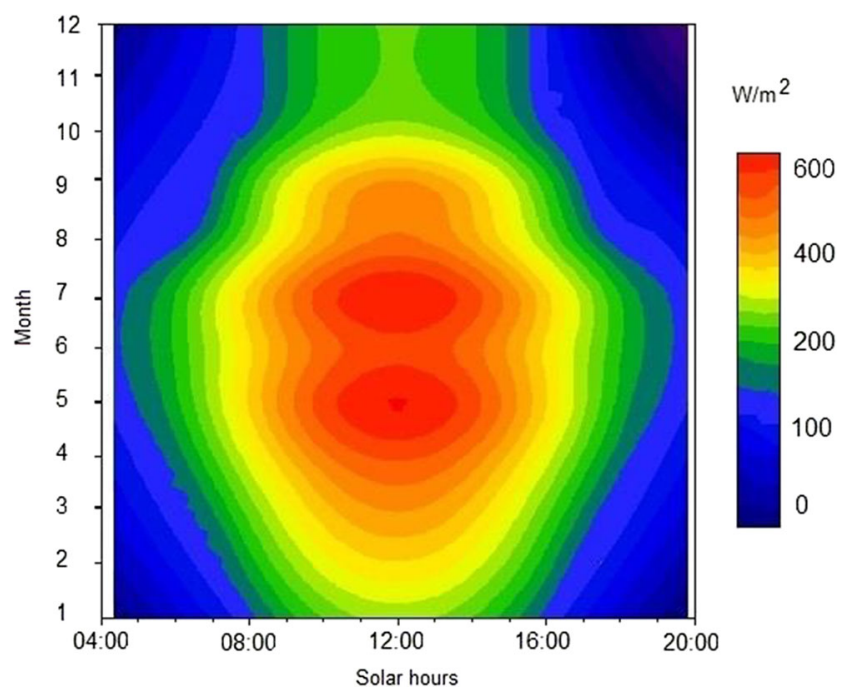

Fig. 1 A 24-hour average distribution of solar radiation intensity for Opole (southwestern part of Poland)
Nevertheless, sewage sludge in which energy characteristics is too low may be used together with other types of waste and include other fuel granulates with appropriate energy and physical parameters.

No information has been found either in the available literature or in the market about solar greenhouse drying of granulated biofuels. Attempts have been made to solar dry coffee pulp (Cubero et al. 2014) for pellet production in open area conditions or olive waste (Maragkaki et al. 2016), wood chips (Perea-Moreno et al. 2016), and RDF fuel (Trojanowska 2016) in the solar greenhouse dryers. However, the wastes were dried in a shredded form. To this end, attempts have been made to develop a new technology of drying granulated biofuels made from sewage sludge, a process requiring designing a new mixing system and defining proper conditions for handling the drying process.

The paper presents research on the determination of optimal solar-drying conditions for granulated biofuels from sewage sludge using the proprietary technology of mixing in a solar dryer.

\section{Materials and methods}

\section{Materials}

Feedstocks obtained from municipal sewage sludge and other waste, such as coal slime, meat and bone meal, and sawdust, have been solar-dried. The method of biofuels production depends on mixing the components in specific proportions then forming granulate in the rotary drum. The drum is equipped with a feeder system ensuring the granulate diameter in the range from 15 to $35 \mathrm{~mm}$ (Wzorek 2008).

The following biofuel compositions were tested:

- 60 wt. $\%$ of sewage sludge, 34 wt. $\%$ coal slime, and 6 wt.\% of quicklime - PBS,

- 75 wt. $\%$ of sewage sludge, 24 wt. $\%$ of meat and bone meal, 1 wt.\% of quicklime - PBM,

- 80 wt. $\%$ of sewage sludge, 19 wt. $\%$ of sawdust and 1wt.\% of quicklime - PBT.

Table 1 shows the biofuel parameters. Following the mixing and granulation operations, biofuels contain between 43 and $68 \%$ of moisture.

In order to define how the granulate size affects the drying rate, biofuels with $15-\mathrm{mm}$ and $35-\mathrm{mm}$ granulations were tested.

\section{Methods}

Biofuels were dried in the solar dryer with a total area of 15 $\mathrm{m}^{2}$, as shown in Fig. 2 . 
Table 1 Properties of biofuels from sewage sludge

\begin{tabular}{lllll}
\hline Parameter & Unit & PBS & PBM & PBT \\
\hline Higher heating value, HHV & MJ/kg & 21.71 & 15.97 & 15.54 \\
The initial moisture content & $\%$ & $43-49$ & $49-54$ & $60-68$ \\
Voltaire matter $^{1}$ & $\%$ d.m. & 34.44 & 55.29 & 59.87 \\
Ash $^{1}$ & $\%$ d.m. & 27.26 & 33.72 & 20.36 \\
\hline
\end{tabular}

d.m. dry mass

${ }^{1}$ Source: Wzorek 2020

The solar dryer was located in Opole, southwestern part of Poland ( $37^{\circ} 51^{\prime} \mathrm{N}$ latitude, $27^{\circ} 51^{\prime} 66 \mathrm{E}$ longitude) in the Opole University of Technology campus.

The drying plant structure (1) was coated with 5-mm thick multi-chamber polycarbonate (2). The plant features two automatic sensors recording both the air temperature and humidity. The solar dryer was also equipped with an air exhaust system comprising six forced draft fans (3) with $624 \mathrm{~m}^{3} / \mathrm{h}$ capacity and two exhaust fans (14) with $95 \mathrm{~m}^{3} / \mathrm{h}$ capacity. The forced draft fans were mounted in two rows in the upper part of the drying plant, while the exhaust fans were installed on the front wall. The forced draft fans are designed to circulate the air inside the dryer in order to improve mass and heat exchange, whereas the exhaust fans are designed to expel humid air from inside the dryer. The air humidity inside the greenhouse was monitored using a hygrometer. Once the designed relative humidity level $(75 \%)$ was exceeded, the ventilation flaps would open and the draft fans start working.

A mixing device was installed in the solar dryer (both the device itself and the manner of mixing are protected under a patent (Wzorek and Głowacki 2014). The device consists of a moving beam (11) with 15 vertical paddle mixers mounted thereon (6); they are shaped so as to lift the material being dried. The mixers are arranged in two rows so as to cover the entire working area. Both the rotation speed of the vertical mixers and the travel speed of the traveling beam are adjustable with a frequency converter. Research into solar drying of biofuels produced from sewage sludge was conducted under various weather conditions, in spring, summer, and the fall. Based on the experience of the existing sewage sludge solar greenhouse dryers, which do not operate in winter time, no drying was performed during that time.

During summer, a period most suitable for determining the best parameters for solar drying, testing was carried out on biofuel beds of varying thickness: 5,10 , and $20 \mathrm{~cm}$, alternating the mixing speed, namely option I no mixing, option II mixing 3 times/day, and option III mixing 5 times/day.

Testing of 35-mm granulated biofuel carried out in the fall and spring involved defining fixed operation of the mixing device with the beam traveling along the entire dryer length 3 times per day of drying, and the vertical mixer rotational speed of $15 \mathrm{rpm}$.

Biofuel samples were taken every day at $6 \mathrm{PM}$ to determine fuel moisture reduction. Moisture levels were measured as stipulated in the PN-80 G-04511 standard. The mixer was not activated on the first day of testing and mixing started only once the fuel top layer had dried up.

Biofuel moisture variations over time were expressed approximately with the following regression function:

$f(X, P)=W(t)=a e^{-b t}$

where $W(t)$ is the moisture variation as a function of time, \%; $a_{i}, b_{i}$, and $b_{i}$ estimates of the regression function structural parameters; and $t$ time, day.

\section{Results and discussion}

Biofuels were solar-dried at various weather conditions. A series of tests carried out in summer demonstrates the most
Fig. 2 Solar greenhouse dryer scheme: 1 drying plant structure, 2 polycarbonate coating, 3 forced draft fans, 4 traveling beam drive, 5 mixer paddle drive, 6 rotating vertical paddles, 7 drying area, 8 concrete floor, 9 external structure of the mixing device, 10 tracks, 11 beam, 12 air temperature and humidity sensor, 13 exhaust flaps, 14 exhaust fans, and 15 entrance to greenhouse dryer
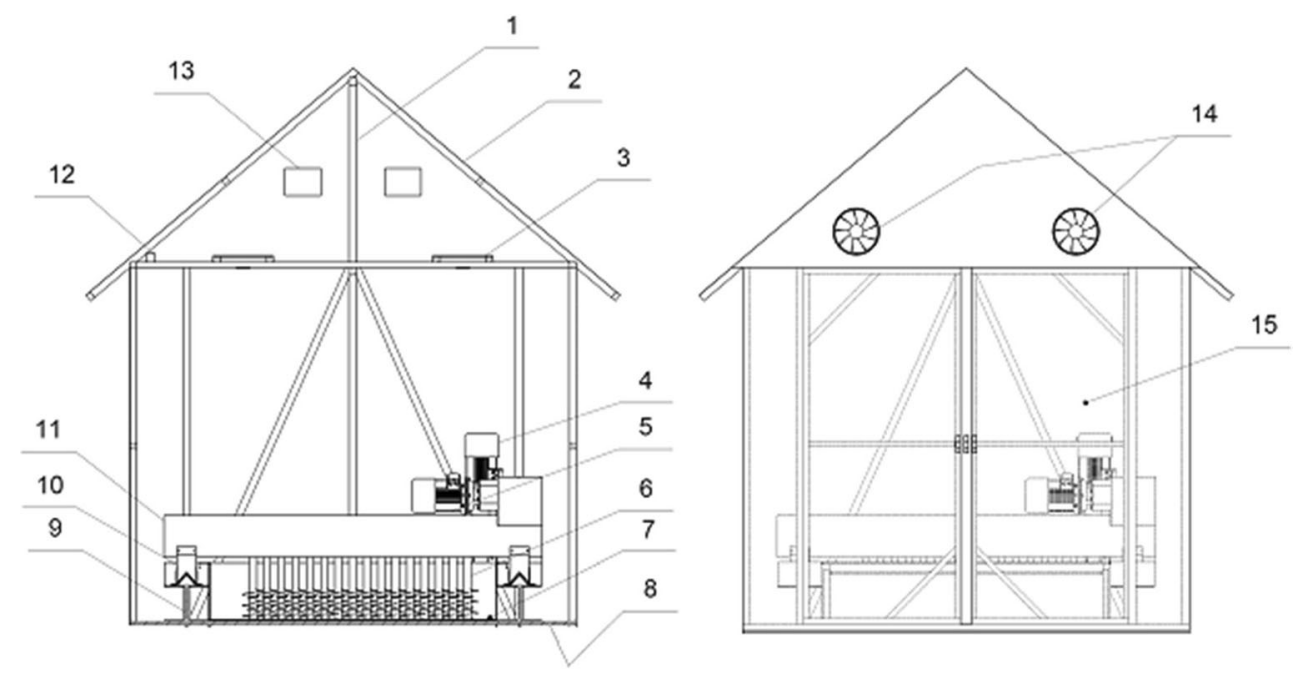
advantageous conditions for solar drying. In Figs. 3 and 4 are presented weather parameters during the summer tests, and Figs. 5, 6, and 7 show results of solar drying in these conditions.

The mean daily temperature of the drying plant air during that time was $31^{\circ} \mathrm{C}$, the mean temperature between $6 \mathrm{AM}$ and 8 PM stood at $41^{\circ} \mathrm{C}$, and between 8 PM and $6 \mathrm{AM}$, it dropped to $21^{\circ} \mathrm{C}$. The total mean daily radiation intensity for the period amounted to $241 \mathrm{~W} / \mathrm{m}^{2}$ and to $340 \mathrm{~W} / \mathrm{m}^{2}$ during the day. Figure 3 illustrates the temperatures and relative humidity of the air inside the solar-drying plant in summer while the total solar radiation is presented in Fig. 4.

Figure 5 illustrates the process of drying one of the biofuels, i.e., PBT which is characteristic for its highest initial moisture content of $65 \%$, with $15-\mathrm{mm}$ and $35-\mathrm{mm}$ granules dried in layers $5-\mathrm{cm}$ and $20-\mathrm{cm}$ thick during summer.

The drying of PBT biofuel demonstrates that mixing it considerably affects the drying rate. Mixing biofuels with various granulations reduced the drying time by 5 days on the average. When using a thicker layer- $20 \mathrm{~cm}$ - differences in the drying time of individual biofuels were observed. It took the PBT fuel with $15-\mathrm{mm}$ granules 8 days to reach $10 \%$ moisture content; while in the case of PBS and PBM, the time required was 7 days.

A similar effect was observed by Krawczyk (2019) for sewage sludge drying who found that adequate sludge bulk mixing proves more efficient than operation at a reduced sludge layer thickness.

It is due to the fact that mixing facilitates elimination of moisture from inside the biofuel layer being dried, raises the fuel granules from the bottom to the top part of the layer, and thus increases the contact between the newly turned layers and the heated air.

It is well-known that the rate of moisture evaporation depends, among others, on the contact surface of the drying medium and the dried material. The more extended the contact surface, the higher the rate of moisture evaporation may be obtained. The phenomenon was described in the literature inter alia by Ruiz et al. (2007), Léonard et al. (2003), and Luboschik (1999).

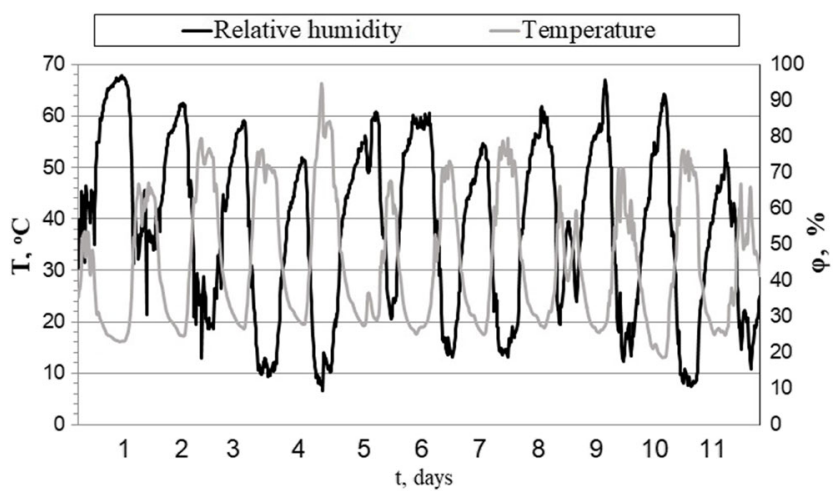

Fig. 3 Relative temperature and humidity of air in the solar dryer in summer test

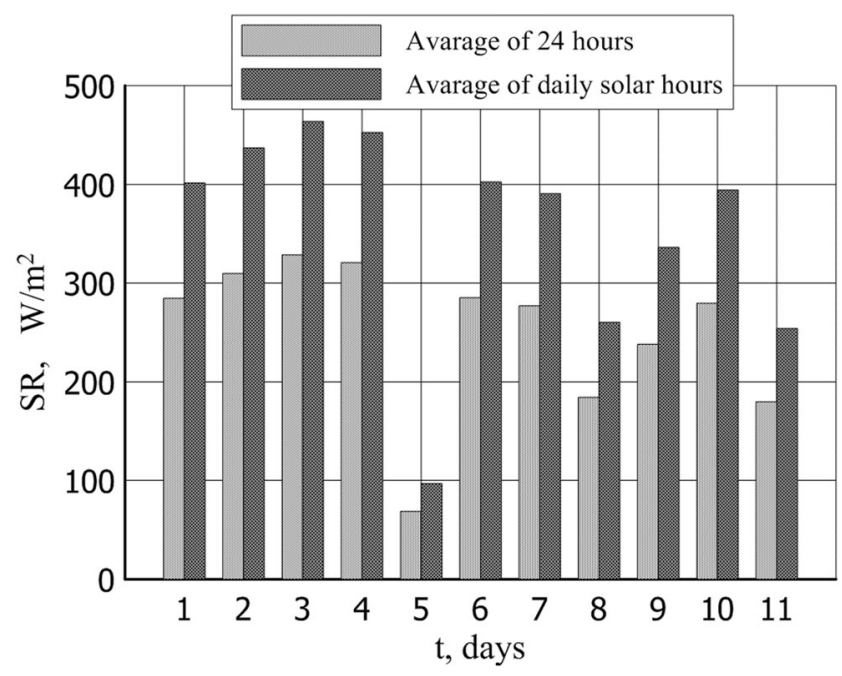

Fig. 4 Total solar radiation (SR) in summer test

The drying rate is also improved when the air inside the solar dryer is mixed by the forced draft fans and circulated by the exhaust fans. Segier and Bux (2006) claim that the air mixing is an order of magnitude less effective (per unit of air discharge) than ventilation.

Comparing the size of granules being dried in various layers, on the other hand, demonstrated that their size affects the drying time to a much lesser degree than the thickness of the layer of the fuel being dried.

To illustrate the point, Fig. 6 shows changes in moisture content of PBT biofuel depending on the size of granules being dried in 5-cm and 10-cm thick layers with mixing 5 times/day.

Ten percent of moisture content for PBT biofuel dried in 5$\mathrm{cm}$ thick layers with 5 times/day mixing was obtained after 6 days. The same result for $10-\mathrm{cm}$ thick layers required 9 days of drying.

The performed tests demonstrate that it is advantageous to dry all biofuels in a 10-cm thick layer which is mixed 5 times per day (Fig. 7).

Figure 7 shows that PBS and PBM biofuels required 6 days to reach the $10 \%$ moisture content when dried under such conditions, while the PBT fuel needed 8 days.

The mixing systems used in solar drying of sewage sludge use various devices, such as the electric mole which continuously moves haphazardly here and there over the material being dried (Thermo-system.Industrie-\&Trocknungstechnik $\mathrm{GmbH}$ ), windrow turners (Veolia 2020), suspended traveling tillers, or selfpropelled rotary turners traveling between one and several times across the dryer (Trojanowska 2016). Depending on the mixing technology, they can handle various layer thicknesses of the material being dried.

From an economic point of view, however, drying as much material per area unit as possible seems the best solution; however, to determine the best conditions for the process, one must also consider the process rate, product quality, and prevention of putrefaction processes. 

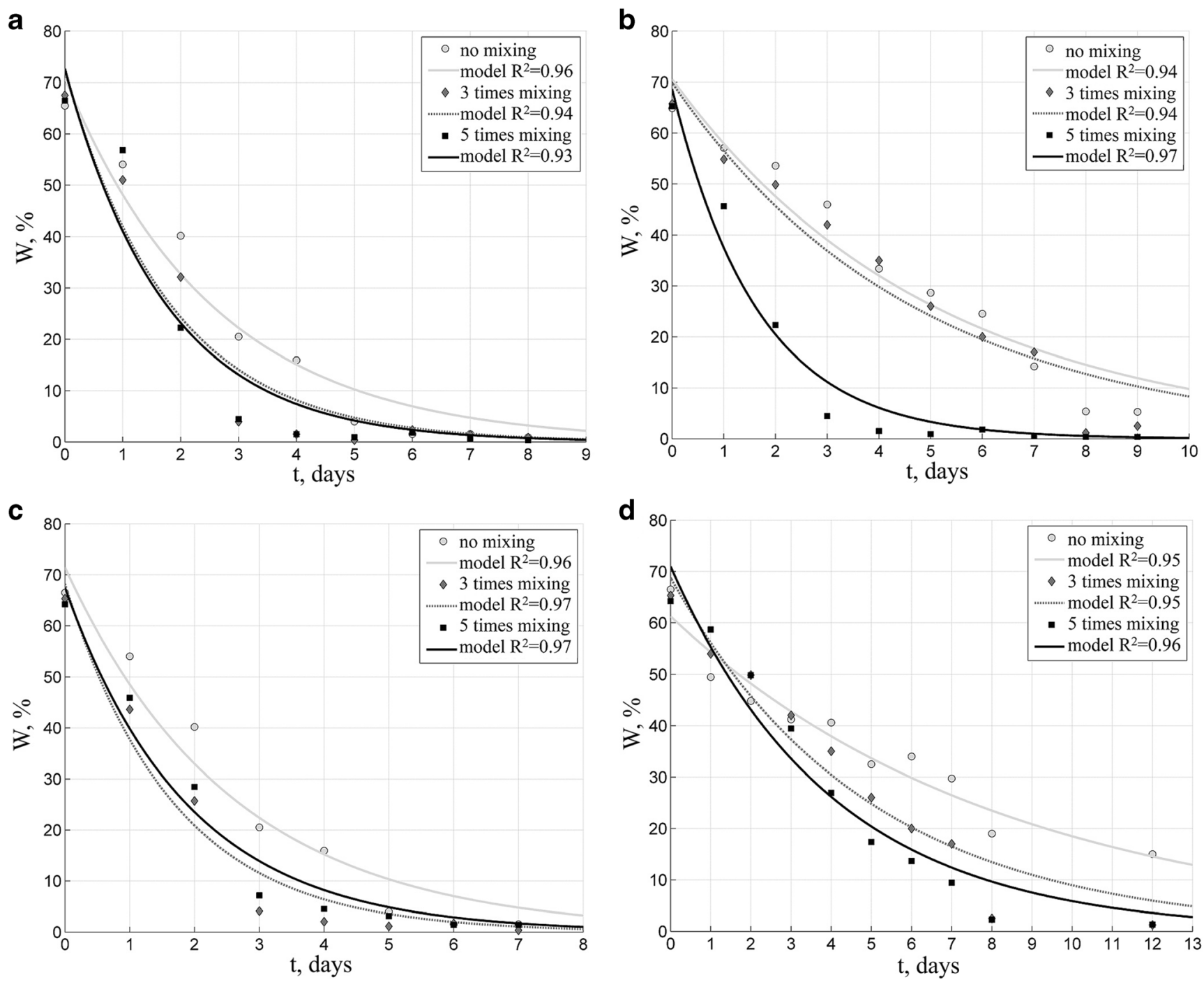

Fig. 5 Changes in moisture content of PBT biofuel (W) during solar drying in summer: (a) 15-mm granules, 5-cm layer; (b) 15-mm granules, 20-cm layer; (c) 35-mm granules, 5-cm layer; (d) 35-mm granules, 20-cm layer

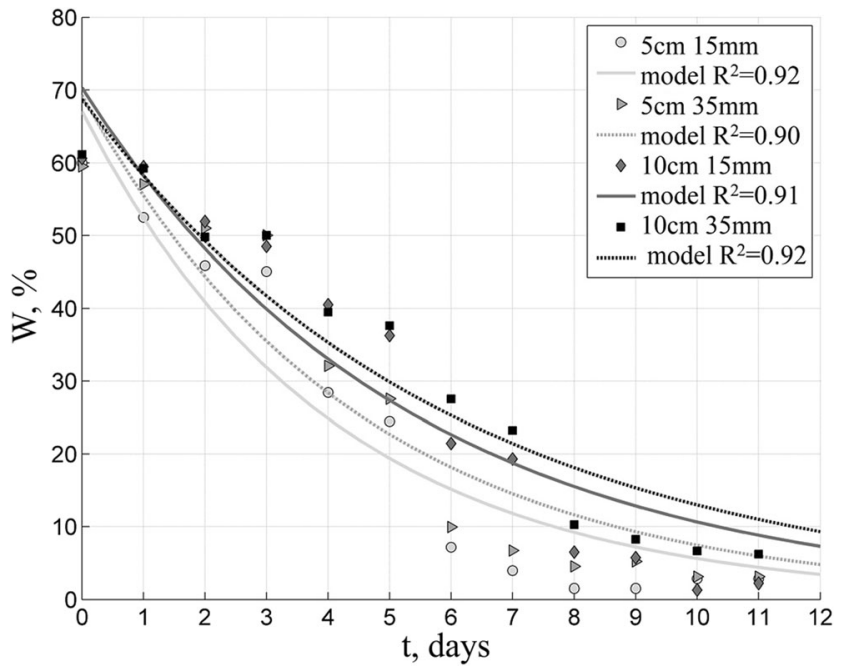

Fig. 6 Changes in moisture content of PBT biofuel with 15-mm and 35mm granules (5- and 10-cm layers) - summer conditions

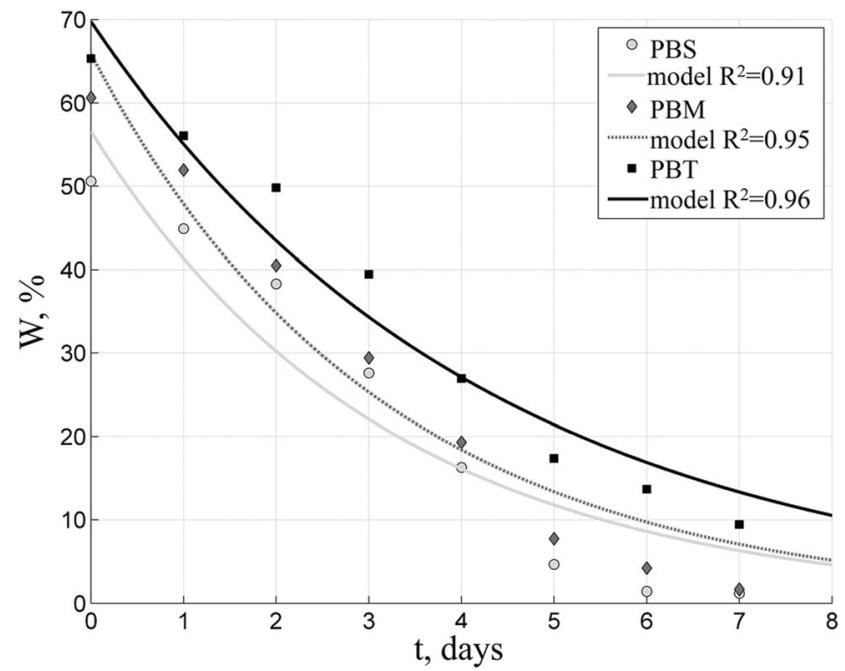

Fig. 7 Changes in moisture content of biofuels with 35-mm granules (10$\mathrm{cm}$ layer) - summer conditions 
Fig. 8 Temperature and relative humidity of the air inside the solar dryer in autumn test

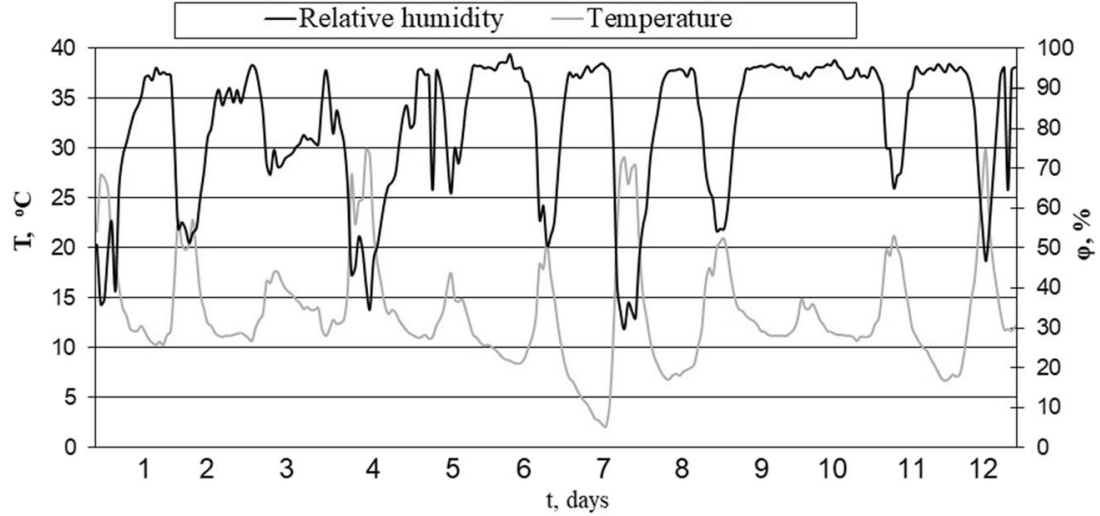

Based on the tests carried out in summer, it was determined that in the case of granulated biofuels, 10-cm thick layers which are mixed 5 times per day give the best results, and tests performed in the fall and spring were designed for exactly such conditions.

Figures 8, 9, 10, and 11 illustrate the results of tests carried out in the fall (September/October). Figure 8 shows the temperature and relative humidity of the air at that time, while Fig. 9 illustrates the total radiation intensity. Throughout the entire test period, the average daytime temperature in the drying plant remained at $17{ }^{\circ} \mathrm{C}$, dropping to $10^{\circ} \mathrm{C}$ at night. Periods of precipitation observed during that time raised the atmospheric air relative humidity to $80 \%$. The mean daily total radiation intensity for the test period amounted to $135 \mathrm{~W} / \mathrm{m}^{2}$ and to $231 \mathrm{~W} / \mathrm{m}^{2}$ during the day.

The curves of drying in autumn (Fig. 10) resemble those obtained in summer, with differences only in the time required to reach the required $10 \%$ moisture content; in our case, the time varied from 9 days for the PBS, to 10 days for the PBM, and more than 11 days for the PBT biofuel.

The drying period in spring was characteristic for the lowest temperature of the air (even some cases of temperatures

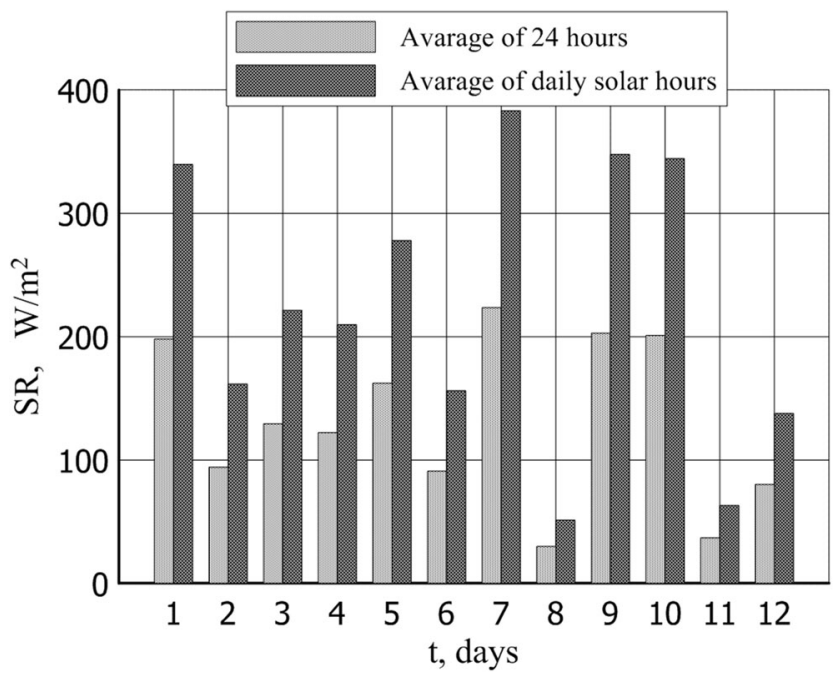

Fig. 9 Total solar radiation (SR) in autumn test below freezing were observed) and the lowest total radiation intensity. The average air temperature inside the drying plant over that period stood at $12{ }^{\circ} \mathrm{C}$ with $69 \%$ relative humidity. The mean daily total radiation intensity for the investigated period amounted to $86 \mathrm{~W} / \mathrm{m}^{2}$ and $171 \mathrm{~W} / \mathrm{m}^{2}$ during the day. Figure 11 presents the drying results obtained in the spring (March/April).

The spring when the tests were run was unusually chilly, with the lowest air temperatures observed (some of them even below freezing) and the lowest total radiation intensity. Just like during the previous tests, the PBS biofuel demonstrated the fastest moisture reduction, and compared to the fall season, the fuel drying time took longer. The PBS biofuel required 8 days to bring the moisture below $10 \%$, while both the PBM and PBT biofuels took 14 days to do the same. Similar results were obtained when bio-drying sewage sludge: Velis et al. (2009) reported the sludge reached $20 \%$ moisture within $7-$ 15 days, while according to Tun and Juchelková (2019), drying municipal solid waste (MSW) in a solar-drying plant may take even up to 38 days at the indoor temperature of $58^{\circ} \mathrm{C}$.

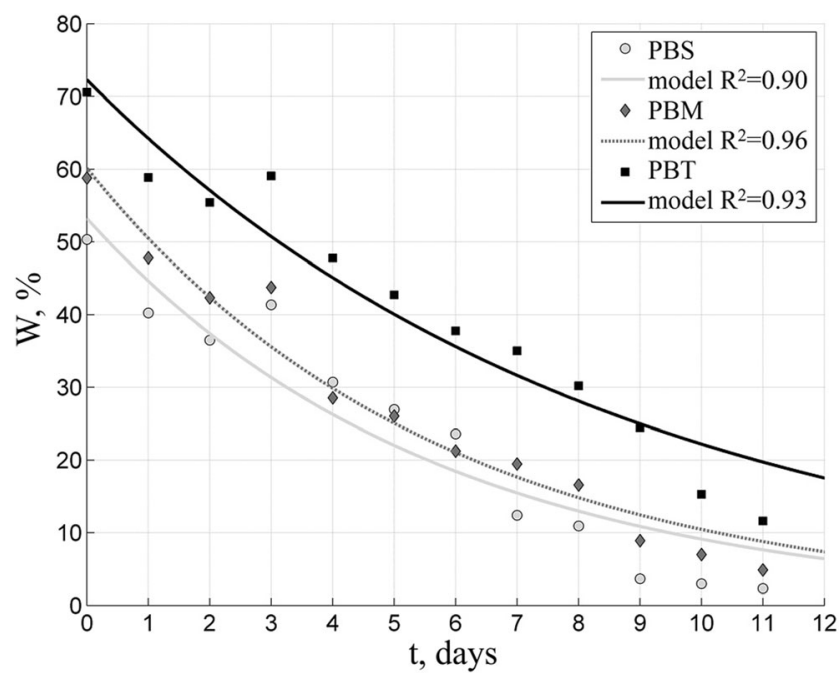

Fig. 10 Changes in moisture content of biofuels with $35 \mathrm{~mm}$ dried in a 10 -cm thick layer in autumn test 


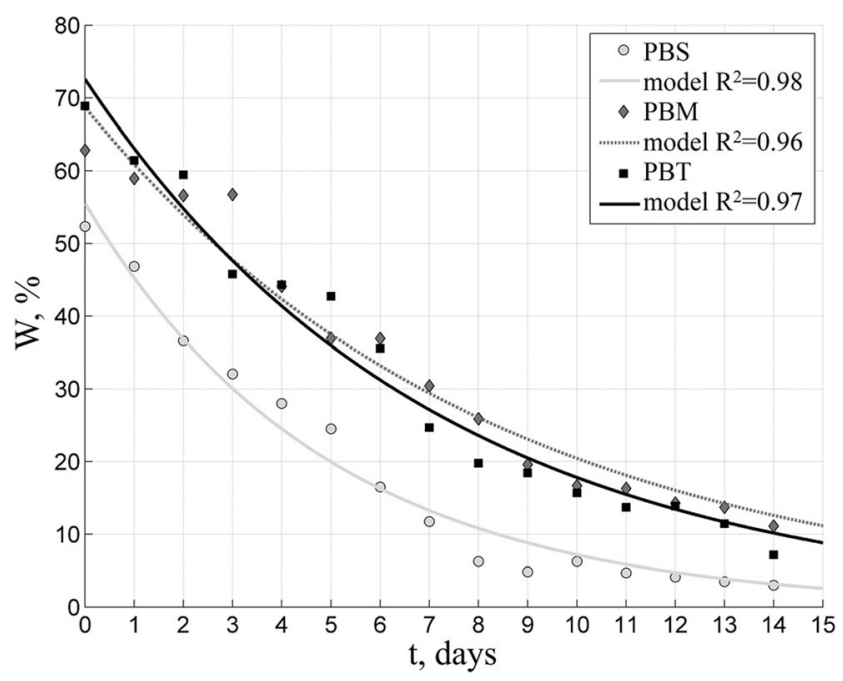

Fig. 11 Changes in moisture content of biofuels with $35 \mathrm{~mm}$ dried in a 10 -cm thick layer in spring test

The drying time for sewage sludge alone may also last from a few to more than 30 days. For example, BoguniewiczZablocka et al. (2020) report solar drying of municipal sewage sludge containing $88 \%$ water and arranged in 5-mm layer under the Polish weather conditions and with outdoor temperature $<20 \mathrm{C}$ takes roughly 4 weeks.

According to Socias (2011), Luboschik (1999), and ElAriny and Miller (1984) carrying out the research under various climate conditions, it can be concluded that the average daily loss of moisture may ranges from 2 to $17 \mathrm{~kg} \mathrm{H} \mathrm{H}_{2} \mathrm{O} / \mathrm{m}^{2}$.

Sobczyk and Sypuła (2011) reports that drying sewage sludge in solar dryers under the Polish weather conditions requires approximately $17-20 \mathrm{~kW}_{\mathrm{t}} / \mathrm{Mg}$ of dehydrated sewage sludge, whereas hybrid plants using additional energy require 88-305 kW/Mg for systems featuring a heat pump, and 108$388 \mathrm{~kW} / \mathrm{Mg}$ for dryers using a heating floor in winter, or roughly $4 \%$ of a thermal dryer costs. In the case of biofuels based on sewage sludge, one may assume operating costs and expenses to be comparable to those of typical solar drying of sewage sludge.

The originally designed mixing system together with appropriate operating parameters of the equipment (vertical paddle mixer rpms and turning frequency) does not affect the granule structure negatively. No granule cracking or crumbling has been observed. It may be stated unequivocally that mixing not only accelerates the entire process but also - at the initial drying period when granules still remain plastic-it prevents their sticking and clumping into large agglomerates.

Table 2 Lower heating value of biofuels from sewage sludge

\begin{tabular}{lllll}
\hline Parameter & Unit & PBS & PBM & PBT \\
\hline Lower heating value (LHV)* & MJ/kg & 18.83 & 13.09 & 13.74
\end{tabular}

*Calculated: moisture - 10\%; hydrogen - $3.91 \%$ d.m. (PBS); $4.12 \%$ d.m. (PBM); $4.43 \%$ d.m. (PBT)
The main purpose of mixing sewage sludge with other components is to increase the energy value and reduce initial moisture of sewage sludge. It is well-known that moisture is a ballast and decreases the fuel energy utility.

Biofuels dried to content of moisture about $10 \%$ have lower heating value in the range of 13-19 MJ/kg (Table 2).

Biosolid fuels have typically a value of LHV like woody and agricultural biomass, which may be in the range of 13-22 $\mathrm{MJ} / \mathrm{kg}$ (Demirbas et al. 2006). Researches presented by other authors show similar level of calorific value of fuels from sewage sludge compering to tested biofuels. According to Jiang et al. (2016), pellets made from sewage sludge and rice straw have calorific value of $15 \mathrm{MJ} / \mathrm{kg}$ and from sewage sludge and unclassified oats above $14 \mathrm{MJ} / \mathrm{kg}$ (Junga et al. 2017).

Valorization of physical and energy properties of sewage sludge increases the chances of their use in energy processes.

\section{Conclusions}

Solar drying seems to be an economical solution for pretreatment of highly hydrated wastes for further thermal utilization because requires very low-energy input and allows to obtain the appropriate properties of feedstocks which are favorable for the combustion and transport.

The method for production of various types of biofuels from sewage sludge, such as sewage sludge with coal slimePBS, sewage sludge with meat and bone meal-PBM, or sewage sludge with sawdust-PBT, makes it possible to choose the type of biofuel to be produced depending on locally available waste.

Based on the tests performed, optimum conditions for the drying process utilizing solar energy were defined for each biofuel, including, especially, the fuel layer thickness plus the method and intensity of the mixing.

It was found that the granule size affected the drying efficiency only to a negligible extent. Another conclusion reached was that the drying process was more effective when the fuel layer was $10 \mathrm{~cm}$ thick. It might be expected that assuming the same layer thickness, the water content of the PBS and PBM biofuels would drop below $10 \%$ after 8 days both in spring and in fall. During summer, the same result might be achieved for all the biofuels typically after 4 days.

Tests show that winter drying of biofuels is possible only when an additional source of heat is used to support the operation of the solar dryer, such as waste heat of industrial processes or biogas produced in wastewater treatment plants.

In summary, tests support the assertion that an original method for solar drying of biofuels based on sewage sludge and other waste has been developed and may be successfully applied in wastewater treatment plants which process waste into fuels, without incurring any additional costs for the heat 
supply, as is the case with thermal drying. The method makes it possible to use sewage sludge with a low net calorific value. Mixing it with other appropriately selected components allows to prepare a biofuel suitable for applications for example in cement clinker production.

Author contribution The manuscript was entirely written by MW.

Funding This study was funded by Opole University of Technology.

Data availability Not applicable

\section{Declarations}

\section{Ethics approval and consent to participate Not applicable}

\section{Consent for publication Not applicable}

Competing interests The authors declare no competing interests.

Open Access This article is licensed under a Creative Commons Attribution 4.0 International License, which permits use, sharing, adaptation, distribution and reproduction in any medium or format, as long as you give appropriate credit to the original author(s) and the source, provide a link to the Creative Commons licence, and indicate if changes were made. The images or other third party material in this article are included in the article's Creative Commons licence, unless indicated otherwise in a credit line to the material. If material is not included in the article's Creative Commons licence and your intended use is not permitted by statutory regulation or exceeds the permitted use, you will need to obtain permission directly from the copyright holder. To view a copy of this licence, visit http://creativecommons.org/licenses/by/4.0/.

\section{References}

Ab Jalil NA, Basri H, Ahmad Basri NE, Abushammala MFM (2016) Biodrying of municipal solid waste under different ventilation periods. Environ Eng Res 21:145-151

Alamia A, Ström H, Thunman H (2015) Design of an integrated dryer and conveyor belt for woody biofuels. Biomass Bioenerg 77:92-109

Bennamoun L (2012) Solar drying of wastewater sludge: a review. Renew Sust Energ Rev 16:1061-1073

Bennamoun L, Arlabosse P, Léonard A (2013) Review on fundamental aspect of application of drying process to wastewater sludge. Renewable and Sustainable Energy Reviews 28:29-43

Boguniewicz-Zablocka J, Klosok-Bazan I, Capodaglio AG (2020) Sustainable management of biological solids in small treatment plants: overview of strategies and reuse options for a solar drying facility in Poland. Environ Sci Pollut Res. https://doi.org/10.1007/ s11356-020-10200-9

Bożym M, Bok A (2015) Advantages and disadvantages of the solar drying of sewage sludge in Poland. Technical Transactions Environ Eng 12:171-179

Callegari A, Bolognesi S, Cecconet D (2020) Capodaglio AG (2020) Production technologies, current role, and future prospects of biofuels feedstocks: a state-of-the-art review. Crit Rev Env Sci Tec 50(4):384-436
Campean M, Marinescu I (2011) Solar systems for wood drying. Environ Eng Manag J 10(8):1069-1076

Capodaglio AG, Olsson G (2020) Energy issues in sustainable urban wastewater management: use, demand reduction and recovery in the urban water cycle. Sustainability (Switzerland) 12(1):266

Capodaglio AG, Callegari A, Dondi D (2016) Microwave-induced pyrolysis for production of sustainable biodiesel from waste sludges. Waste Biomass Valori 7(4):703-709

Collard M, Teychene B, Lemee L (2017) Comparison of three different wastewater sludge and their respective drying processes: solar, thermal and reed beds. Impact on organic matter characteristics. J Environ Manage 203:760-767

Cubero R, Roque RM, Volaret J, Filho MT (2014) Use of coffee (coffea arabica) pulp for the production of briquettes and pellets for heat generation. Ciência e Agrotecnologia 38(5):461-470

Demirbas A, Pehlivan E, Altun T (2006) Potential evolution of Turkish agricultural residues as biogas, biochar and biooil sources. Int $\mathrm{J}$ Hydrog Energy 31(5):613-620

El-Ariny S, Miller HI (1984) Utilization of solar energy for sludge drying beds. J Solar Energ Eng 106:351-357

EU Hub. The European Commission's science and knowledge service http://re.jrc.ec.europa.eu/pvgis/apps4/pvest.php\# (Accessed on 20 September 2020)

Fonts I, Gea G, Azuara M, Ábrego J, Arauzo J (2012) Sewage sludge pyrolysis for liquid production: a review. Renew Sust Energy Rev 16:2781-2805

Helwa NH, Khater HA, Enayet MN, Hashish MI (2004) Experimental evaluation of solar kiln for drying wood. Dry Technol 22(4):703717

Hii CL, Jangam SV, Ong SP, Mujumdar AS (eds) (2012) Solar drying: fundamentals, applications and innovations. TPR Group Publication, ISBN: 978-981-07-3336-0

IST-Anlagenbau GmbH http://www.wendewolf.com (Accessed on 20 September 2020)

Jiang L, Yuan X, Xiao Z, Liang J, Li H, Cao L, Wang H, Chen X, Zeng G (2016) A comparative study of biomass pellet and biomass-sludge mixed pellet: energy input and pellet properties. Energy Convers Manag 126:509-515

Junga R, Wzorek M, Kaszubska M (2017) Technical and environmental performance of $10 \mathrm{~kW}$ understocker boiler during combustion of biomass and conventional fuels. E3S Web of Conferences 19: 01009. https://doi.org/10.1051/e3sconf/20171901009

Krawczyk P (2019) The relative change of drying rate as a function of the cumulative ventilation air drying potential in a thin-layer solar drying facility. IOP Conf Ser Mater Sci Eng 556:012055

Léonard A, Blacher S, Pirard R, Marchot P, Pirard JP, Crine M (2003) Multiscale texture characterization of wastewater sludges dried in a convective rig. Dry Technol 21:1507-1526

Luboschik U (1999) Solar sludge drying-based on the IST process. Renew Energy 16:785-788

Mailler R, Gasperi J, Chebbo G, Rocher V (2014) Priority and emerging pollutants in sewage sludge and fate during sludge treatment. Waste Manage 34:1217-1226

Maragkaki A, Gallio F, Sabathianakis T, Sompanidis C, Lolos G, Mayrogiannis G, Koukakis G, Lasaridi K, Manios T (2016) Initial investigation of the solar drying method for the drying of olive oil by-products. Waste and Biomass Valorization 7:819-830

Maurer C, Müller J (2019) Drying characteristics of biogas digestate in a hybrid waste-heat/solar dryer. Energies 12:1294

Mehrdadi N, Joshi SG, Nasrabadi T, Hoveidi H (2007) Application of solar energy for drying of sludge from pharmaceutical industrial wastewater and probable reuse. Int J Environ Res 1:42-48

Murakami T, Suzuki Y, Nagasawa H, Yamamoto T, Koseki T, Hirose H (2009) Combustion characteristics of sewage sludge in an incineration plant for energy recovery. Fuel Process Technol 90:778-783 
Oladejo J, Shi K, Luo X, Yang G, Wu T (2019) A review of sludge-toenergy recovery methods. Energies 12:60-98

Ozaki N, Nakazato A, Nakashima K, Kindaichi T, Ohashi A (2017) Loading and removal of PAHs, fragrance compounds, triclosan and toxicity by composting process from sewage sludge. Sci Total Environ 605-606:860-866

Pawlita-Posmyk M, Wzorek M (2016) Analysis of domestic sewage treatment system. Chemik 70(10):623-625

Pawlita-Posmyk M, Wzorek M (2017) Assessment of application of selected waste for production of biogas. E3S Web of Conferences 19: 02017. https://doi.org/10.1051/e3sconf/20171902017

Perazzini H, Freire FB, Freire FB, Freire JT (2016) Thermal treatment of solid wastes using drying technologies: a review. Dry Technol 34: $39-52$

Perea-Moreno AJ, Juaidi A, Manzano-Agugliaro F (2016) Solar greenhouse dryer system for wood chips improvement as biofuel. J Clean Prod 135:1233-1241

Poland's energy policy until 2030 (2009) Ministry of Economy. Warsaw, November 10

Raboni M, Viotti P, Capodaglio AG (2015) A comprehensive analysis of the current and future role of biofuels for transport in the European Union (EU). Revista Ambiente e Agua 10(1):9-21

Raheem A, Sikarwar VS, He J, Dastyar W, Dionysiou DD, Wang W, Zhao M (2018) Opportunities and challenges in sustainable treatment and resource reuse of sewage sludge: a review. Chem Eng J 337:616-641

Ruiz T, Wisniewski C, Kaosol T, Persin F (2007) Influence of organic content in dewatering and shrinkage of urban residual sludge under controlled atmospheric drying. Transactions of IChemE Part B: Process Saf Environ 85:104-110

Segier I, Bux M (2006) Modeling solar drying rate of wastewater sludge. Dry Technol 11(24):1353-1363

Shin Y, Kim H, Chun H (2000) Drying of water treatment process sludge in a fluidised bed dryer. Korean J Chem Eng 17(12000): 22

Singh P, Shrivastavaa V, Kuma A (2018) Recent developments in greenhouse solar drying: a review. Renew Sust Energ Rev 82:3250-3262

Sobczyk R, Sypuła M (2011) Doświadczenia z eksploatacji suszarni osadów na oczyszczalni ścieków w Kłodzku, Forum Eksploatatora, Nr 2:14-18

Socias I (2011) The solar drying plant in Mallorca: the drying process in waste management. Proceedings of the III European Drying Conference - EuroDrying' 2011, 26-28 October, 1-3 Palma de Mallorca, Spain

Statistics Poland (2018) Statistical information and studies. http://stat. gov.pl (Accessed 20 September 2020)
Tańczuk M, Kostowski W (2021) Technical, energetic and economic optimization analysis of selection of heat source for municipal sewage sludge dryer. Energies 14(2):316

Thermo-system. Industrie- \& Trocknungstechnik GmbH https://www. thermo-system.com (Accessed on 20 September 2020)

Trojanowska K (2016) Ekonomika słonecznych suszarni osadów ściekowych - przegląd pierwszej dekady polskich doświadczeń. Forum Eksploatatora 3:56-63

Tun M, Juchelková D (2019) Drying methods for municipal solid waste quality improvement in the developed and developing countries: a review. Environ Eng Res 24(4):529-542

Velis CA, Longhurst PJ, Drew GH, Smith R, Pollard SJT (2009) Biodrying for mechanical-biological treatment of wastes: a review of process science and engineering. Bioresour Technol 100:27472761

Veolia https://www.veoliawatertechnologies.com (Accessed on 20 September 2020)

VijayaVenkataRaman S, Iniyan S, Goic R (2012) A review of solar drying technologies. Renew Sust Energy Rev 16(5): $2652-2670$

Wasielewski R, Stelmach S, Jagustyn B (2013) Sewage sludge as a renewable energy carrier and $\mathrm{CO}_{2}$ zero emission biomass in cocombustion with coal. Environ Prot Eng 39(2):145-152

Werther J, Ogada T (1999) Sewage sludge combustion. Prog Energy Combust Sci 25:55-116

Wzorek M (2008) Physical and chemical properties of fuel containing animal waste. WIT Transactions on Ecology and the Environment 109:69-77

Wzorek M (2020) Evaluating the potential for combustion of biofuels in grate furnaces. Energies 13:1951

Wzorek M, Głowacki T (2014) A device for mixing, especially sewage sludge. Utility model PL W.121829 31 July 2014

Wzorek M, Troniewski L (2007) Application of sewage sludge as a component of alternative fuel, Environmental Engineering Proceedings of the 2nd National Congress of Environmental Engineering, 311-316.

Yoo JY, Kim HJ, Woo EJ, Park CJ (2017) On solar energy utilization for drying technology. Int J Environ Sci Develop 8(4):305-311

Zhang D, Luo W, Yuan J, Guoxue LG (2018) Co-biodrying of sewage sludge and organic fraction of municipal solid waste: Role of mixing proportions. Waste Manage 77:333-340

Publisher's note Springer Nature remains neutral with regard to jurisdictional claims in published maps and institutional affiliations. 